\title{
SASVi - Sistema de Asistencia y Seguridad Vial
}

\author{
Fernando Martínez, Luis C. González, Manuel R. Carlos
}

Universidad Autónoma de Chihuahua, Facultad de Ingeniería, 31125, Chihuahua, MX

\{fmartine, lcgonzalez, p168786\}@uach.mx

\begin{abstract}
Resumen. En los países desarrollados se observa un despliegue a gran escala de tecnología para desarrollar actividades de vigilancia ciudadana y de asistencia vial. Cámaras y redes de sensores dan soporte a estrategias de agilización del tráfico y de contención de delincuencia en las zonas urbanas. En los países en desarrollo, por el contrario, se observa un limitado nivel de infraestructura tecnológica para atender este tipo de problemas sociales. La computación urbana emerge como una opción que permite aprovechar la tecnología disponible en contextos públicos y a través de ésta recolectar información para dar soporte a las estrategias de gobierno en las grandes ciudades. La plataforma de asistencia y seguridad vial propuesta por SASVi busca contribuir en esta línea. SASVi explota las tecnologías de sensores integradas en los dispositivos móviles y utiliza sistemas de almacenamiento en la nube para dar soporte al concepto de informática urbana. Esta plataforma ofrece recursos y facilidades que notifican al conductor acerca de la presencia de irregularidades en el pavimento de avenidas y carreteras, informan acerca de del reglamento vial aplicable a la zona por la cual se transita, y registran evidencia de la interacción entre oficiales de vialidad y conductores.
\end{abstract}

Palabras clave: cómputo móvil, asistencia vial, servicios en la nube.

\section{$1 \quad$ Introducción}

Las grandes urbes enfrentan grandes problemas. Conforme las ciudades se desarrollan, los problemas de congestionamiento de tráfico vehicular, delincuencia y contaminación ambiental se incrementan. En los países desarrollados se observa la integración de plataformas tecnológicas a través de las cuales se puede contar con información que apoya la toma de decisiones en la administración de las ciudades. Redes de cámaras de vigilancia apoyan contextos de seguridad social ya que es posible identificar el comportamiento de individuos. Información proporcionada por redes de sensores ayudan a agilizar el flujo vehicular e incluso habilitan el monitoreo de los niveles de contaminación y de impacto ambiental en diferentes zonas de la ciudad. Las limitaciones tecnológicas que los países en desarrollo presentan, por el contrario, comprometen el aseguramiento de confort y seguridad que los ciudadanos requieren. En estos países la participación de la ciudadanía puede ser el medio para recolectar datos del entorno urbano y recíprocamente recibir mejores servicios por parte del gobierno. Hoy en día los teléfonos celulares cuentan con tecnología de 
sensores GPS, acelerómetro, giróscopo, cámara fotográfica y con canales de comunicación Bluetooth, WiFi y 3G. A través de su teléfono el ciudadano puede escribir una opinión acerca del aspecto que guarda el vecindario en el que vive, puede tomar fotos y utilizarlas como evidencia y a su vez sugerir cambios. La información disponible en las redes sociales da muestra de ello.

La plataforma SASVi, ofrece recursos y facilidades de asistencia y seguridad vial. Los servicios de asistencia incluyen la notificación de irregularidades en el pavimento (e.g. baches y reductores de velocidad sin señalización) de avenidas y carreteras, y la información del reglamento vial aplicable a la zona por la cual se transita. El servicio de seguridad consiste en el registro de evidencia que da soporte a la versión de hechos calificada por el agente vial. Ciudadanía, industria y gobierno pueden verse beneficiadas con este tipo de servicios. Por ejemplo, con los servicios de asistencia los ciudadanos pueden reducir el riesgo de daños a sus vehículos por problemas existentes con el pavimento. La industria podría identificar rutas alternas con mejor flujo vehicular y no comprometer sus tiempos de distribución. El gobierno podría contar con información dura para realizar una mejor calificación del perfil de riesgo de conducción de las personas, y posiblemente mejorar las estrategias de mantenimiento de vías de comunicación terrestres. La plataforma SASVi pretende contribuir con la exploración de soluciones tecnológicas que podrían atender algunas de los retos urbanos del estado de Chihuahua. Chihuahua cuenta con 3.5 millones de habitantes, circulan cerca de 1.5 millones de vehículos, y como en la mayor parte del país se presentan situaciones de conflicto entre ciudadanos y elementos de seguridad vial y policial. Adicionalmente, el clima extremo que prevalece en verano (36-40 grados centígrados) y en invierno (-3-0 grados centígrados) contribuye al deterioro de caminos y carreteras, lo cual también repercute en el nivel de movilidad vehicular.

La organización del trabajo es la siguiente: en la sección 2 se presenta la revisión de algunos de los esfuerzos que la comunidad científica ha realizado para la exploración de soluciones tecnológicas que atiendan factores de movilidad y tráfico urbano así como aquellas propuestas que promueven el uso de la tecnología para brindar asistencia y seguridad a los ciudadanos. En la sección 3, se describe la plataforma tecnológica SASVi y el soporte proporcionada a aspectos de asistencia y seguridad vial. En la sección 4, se ofrecen los resultados del desempeño de SASVi. En la sección 5, se realiza una discusión sobre algunas consideraciones experimentales para SASVi y finalmente, en la sección 6 se ofrecen las conclusiones y el trabajo futuro de este proyecto.

\section{Motivación}

Según información del INEGI, Chihuahua ocupa el tercer lugar a nivel nacional en accidentes de vialidad, de los cuales la mayoría de estos accidentes son causados por conductores que circulan con exceso de velocidad. En el 2012 en el estado de Chihuahua se registraron un total de 30,382 accidentes en la entidad y 17 mil personas perdieron la vida. Por supuesto, en muchos de los casos se observa que el factor humano es relevante [1]. Para contribuir en la reducción de accidentes la comunidad académica ha explorado diferentes niveles de integración de tecnología tanto en el 
auto como en la ciudad. La literatura muestra propuestas con diferente nivel de integración de tecnología enfocadas en asistir a conductores durante su tránsito por las ciudades de forma segura. Por ejemplo, Bierlaire et al. [2] presentan un sistema que hace uso de dispositivos móviles para recabar información de recorridos realizados por los usuarios y con esta información sugiere las mejores rutas para llegar a un lugar desde un sitio determinado en el menor tiempo posible. También se puede utilizar la información geoespacial de la ubicación del automóvil para poder sincronizar servicios de emergencia en caso de encontrarse en zonas desconocidas [3], o en caso de verse involucrado en un accidente vial [4]. A través del teléfono celular que porta la persona es posible incluso identificar su perfil de conducción [5].

Otro de los factores que puede agregar al índice de accidentes es el estado que guarden las carreteras. La administración de estas vías de comunicación puede ser retadora para países que no cuentan con la infraestructura adecuada. La aparición de irregularidades o desperfectos en las carreteras pueden deberse a factores ambientales o humanos. Factores ambientales pueden generar grietas, hundimientos, remoción de tapas de sistemas pluviales y erosión de señalizaciones. Los departamentos que administran las carreteras pueden tener fallas en el mantenimiento de las mismas o la adecuada señalización de por ejemplo reductores de velocidad. Cualquiera que sea su naturaleza, sin embargo, la presencia de estas irregularidades afecta el nivel de movilidad vial. Por lo tanto, resulta importante poder contar con un sistema de identificación de baches, topes y boyas, entre otras irregularidades en los caminos y carreteras para posteriormente contar con un sistema de notificación de la presencia de estos problemas a los conductores. En Kertész et al. [6], por ejemplo, se utilizan dos cámaras y proyectores láser para identificar baches en el pavimento, y con la tecnología Kinect de Microsoft Joubert et al. [7] logra medir la profundidad de los baches. En Mednis et al. [8] se desarrolló la grabación y el análisis de audio proveniente de los baches a través de los cuales se conduce un automóvil. La tecnología de acelerómetros es utilizada por Eriksson et al. [9], quienes clasifican baches, cruces de vías de ferrocarril y reparaciones realizadas a la carretera. Otros autores han aprovechado la disponibilidad de acelerómetros en dispositivos móviles. Fazeen et al. [10], identifica baches y topes a partir de datos provenientes del celular que la persona porta en algún compartimento de su ropa.

Finalmente, existe trabajo que reporta la existencia de conflictos entre elementos que representan la ley y conductores. Por ejemplo, la información que un oficial registra puede entrar en controversia con la declaración de los hechos por parte del conductor [11], lo cual puede generar situaciones ríspidas entre las personas involucradas generando en ocasiones agresiones físicas. Sin embargo, la tecnología portada por un oficial puede agilizar la tarea y agregar veracidad a la generación de reportes, además de reducir riesgos de ataques contra oficiales [12].

\section{Plataforma SASVi}

Como se ha mencionado SASVi pretende ofrecer recursos y facilidades para proveer servicios de asistencia y seguridad vial. El sistema ofrece información referente a la reglamentación de vialidad establecida para diferentes zonas de la ciudad, así como 
notificaciones de las irregularidades presentes en la carretera. Se ofrece adicionalmente, un recurso para registrar audio y video producto de la interacción entre el oficial de vialidad y el conductor. La plataforma tecnológica de SASVi utiliza tecnologías de cómputo móvil y servicios en la nube como se muestra en la figura 1.

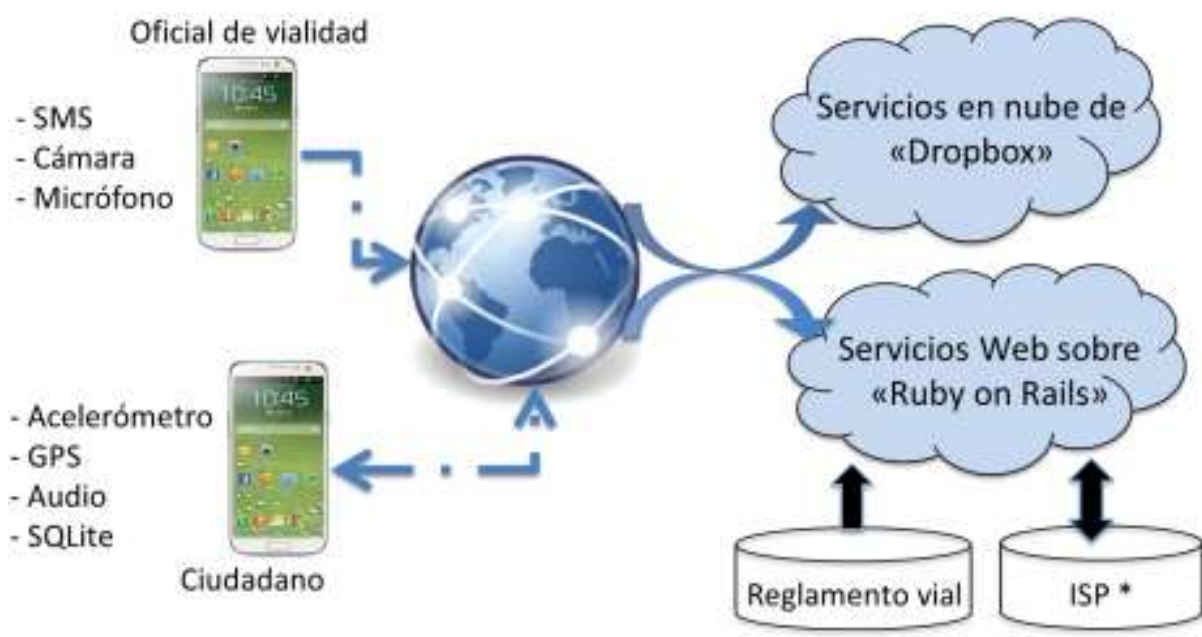

- ISP ["] irregularidades sobre pavimento

Figura 1. El teléfono celular del conductor o del oficial de vialidad representa el nodo de recolección de datos del entorno. Los servicios de asistencia y seguridad se recuperan de la nube.

\subsection{Servicio de Asistencia y Seguridad Vial}

Tanto el conductor como el agente vial utilizan un teléfono inteligente, el cual se comunica con un servidor remoto para gestionar o transferir información según sea el caso. Para el servicio de asistencia vial se hace uso de la posición actual del vehículo y de la orientación del mismo, información que es obtenida a través del GPS y del giroscopio integrados en el teléfono celular. Con esta información se consulta un servidor remoto para actualizar el reglamento vial aplicable a la zona en la cual se transita, y la información de geo-localización disponible para las irregularidades de las carreteras registradas dentro de la misma zona. La información GPS se procesa para calcular la velocidad del automóvil la cual se compara contra las velocidades máximas permitidas. Está información también se utiliza para notificar al conductor de posibles encuentros con baches y/o reductores de velocidad sin la señalización adecuada. SASVi reproduce comandos de voz indicando al conductor cuando se rebasó el límite de velocidad o emite señales sonoras ante la posible presencia de baches, topes u otras irregularidades en el pavimento. Adicionalmente, se realiza un registro local (SQLite) de las faltas cometidas, el cual puede ser revisado 
posteriormente para su análisis. Es posible también configurar esta herramienta para enviar el registro hacia una ubicación remota en caso de que sea necesario un monitoreo en tiempo real.

Para el servicio de seguridad vial se hace uso de la cámara y micrófono disponibles en el teléfono celular. El uso de la cámara depende de la configuración que el usuario elija, pero están disponibles las opciones de captura de imágenes o de video. Adicionalmente, con la activación del servicio de video/foto se activa el recurso "MediaRecorder" que habilita el canal de audio para el micrófono. Para dar soporte al oficial de vialidad, es importante garantizar que se cuenta con al menos una imagen de la persona que se encuentra al frente del volante. Para ello, el sistema se asegura de tomar la foto y almacenarla localmente en el celular. Adicionalmente se genera una copia en escala de grises, la cual es posteriormente binarizada y transferida vía mensaje de texto al centro de mando, con el objetivo de contar con los rasgos del conductor de forma instantánea.

\section{Plataforma informática de SASVi}

En esta sección se describen los diferentes procesos de comunicación que dan soporte a los diferentes niveles de gestión y transferencia de información desarrollados por SASVi, basada en un servicio Web implementado en Ruby on Rails como se observa en la figura 2.

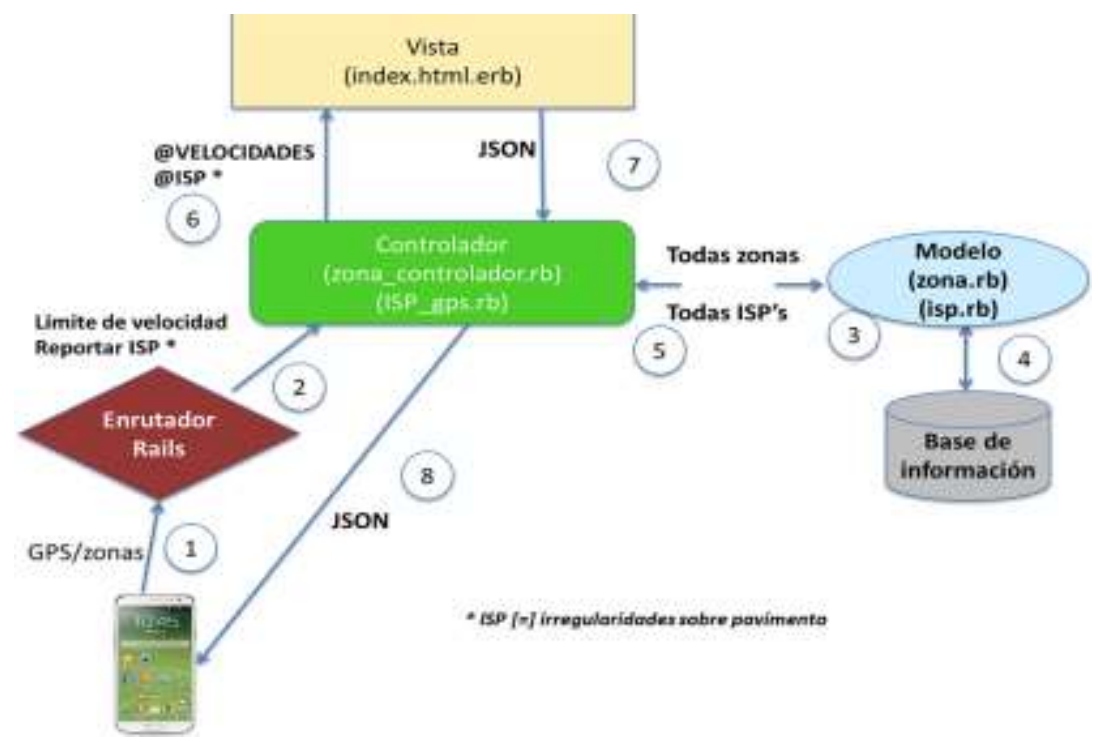

Figura 2. Servicio web en Ruby on Rails utilizado para la gestión de información de SASVi.

El servicio web recibe del dispositivo móvil la petición para la actualización del reglamento vial aplicable a la zona indicada por la información geo-espacial (ubicación del dispositivo móvil) incluida en la petición. Esta conexión al servidor se 
realiza mediante un cliente http el cual envía una petición a la URL del servidor junto con una serie de parámetros incluyendo el nombre de la avenida y la orientación del automóvil, o propiamente dicho del dispositivo móvil:

Private static readonly string ServerURL $=\underline{\text { http://192.168.1.5:3000/speedLimit/Vallarta }}$

Una respuesta en formato JSON es enviada hacia el dispositivo móvil con la información de la reglamentación vial aplicable a la zona, como se muestra en la figura 3.

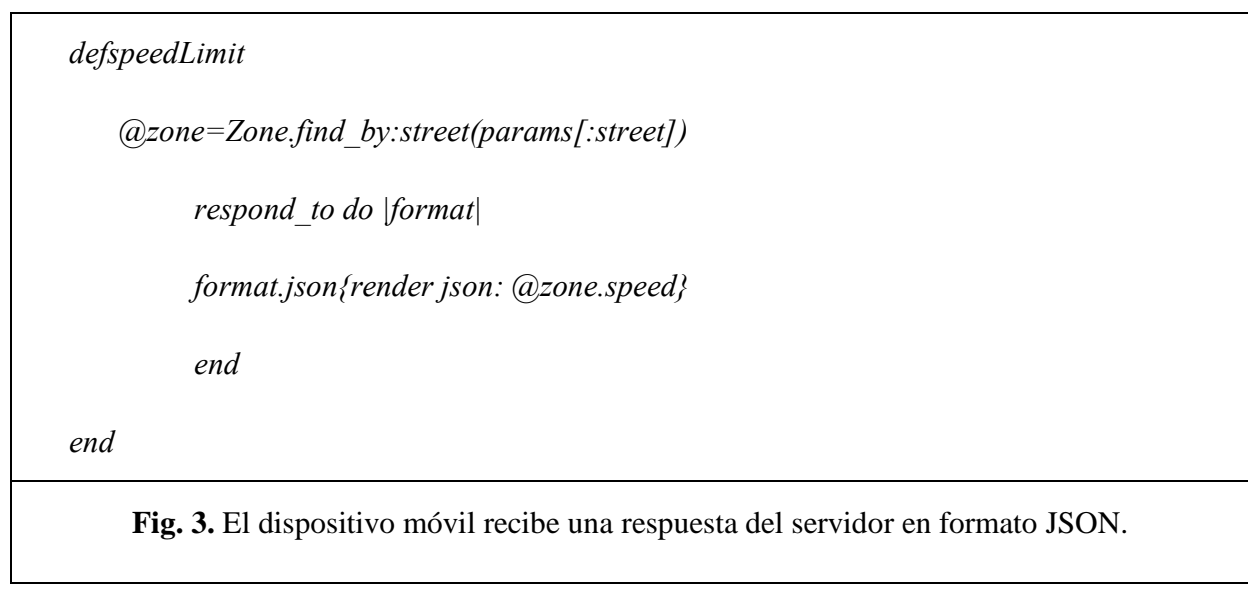

En relación con la transferencia de información que realiza SASVi para brindar soporte al oficial de vialidad, se tiene que el dispositivo móvil establece un canal de comunicación continuo con un servicio en la nube de "Dropbox". Para poder establecer la comunicación entre el celular y Dropbox es necesario que el usuario obtenga una cuenta y por lo tanto las llaves de acceso a este servicio, como se muestra en la figura 4.

AndroidAuthSession session= new AndroidAuthSession(appkeys, ACCES TYPE);

DropboxAPI $<$ AndroidAuthSession $>$ API $=$ new Dropbox $<$ AndroidAuthSession $>$ (session);

API.getSession().startAuthentification(Activity.this);

Fig. 4. Establecimiento de conexión con un servicio en la nube de Dropbox.

Una tarea adicional del sistema informático es la recolección de datos de aceleración proporcionados por el sensor del teléfono celular y la clasificación de irregularidades presentes en el pavimento a lo largo de la carretera. Estos datos son procesados del lado del servidor utilizando una red neuronal "back propagation" [13] con una capa oculta y 5 neuronas. 


\section{Resultados}

La fase de recolección de datos se realizó con teléfonos celulares HTC, con $278 \mathrm{MB}$ de memoria RAM, un solo procesador y una cámara de 5 mega pixeles, durante el verano de 2013 en 2 carros diferentes, un Nissan Sentra 2013 y un Nissan Tsuru 2001. En cada auto se realizaron diversos recorridos cada uno considerando el tránsito sobre avenidas, calles, bulevares y carreteras de la ciudad de Chihuahua, Chihuahua, México. La figura 5 muestra uno de estos recorridos cuya longitud es de 40 Kilómetros.

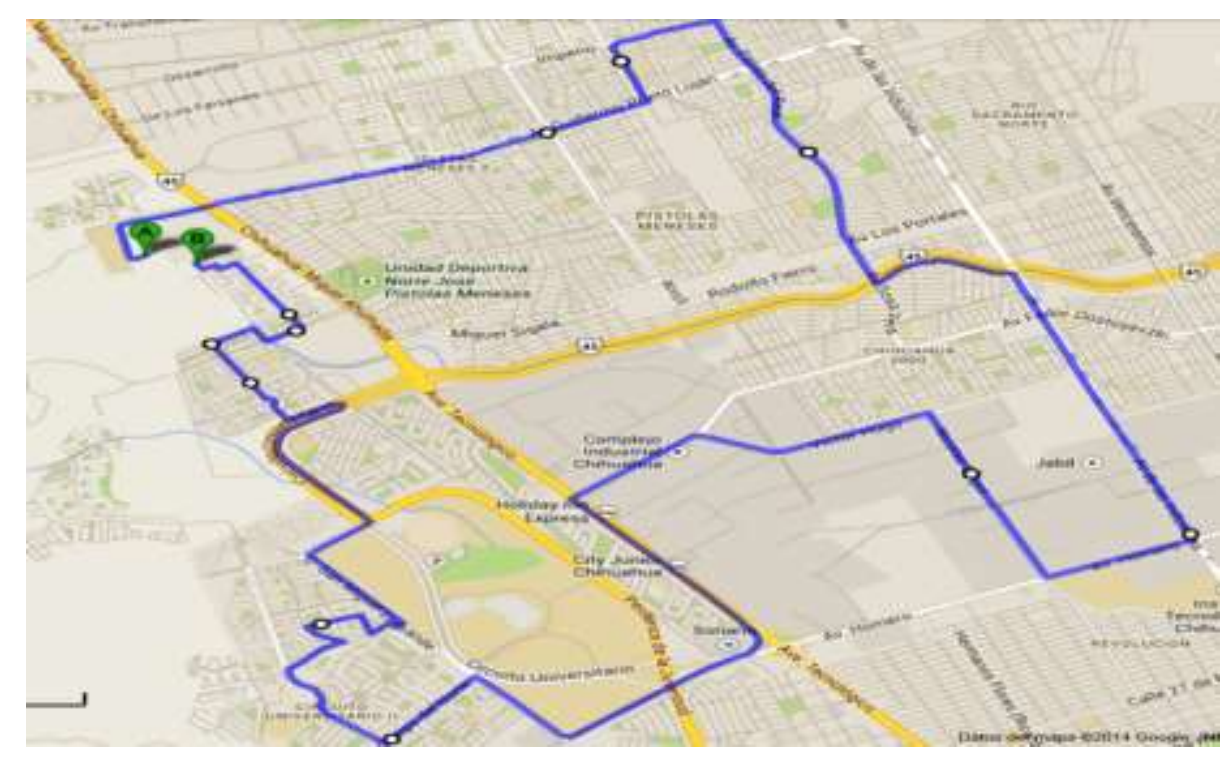

Figura 5. Trayectoria de $40 \mathrm{Km}$. sobre la cual se recolectaron primeramente eventos de aceleración asociados a baches, topes y boyas. Posteriormente se prueban los servicios de asistencia y seguridad que ofrece SASVi.

En este recorrido existen zonas escolares con límites de velocidad de $20 \mathrm{Km} / \mathrm{hr}$. A lo largo de la trayectoria se recolectaron eventos correspondientes a 24 baches, 46 boyas y 51 topes. La red neuronal implementada del lado del servidor clasificó con un porcentaje de acierto superior al $85 \%$ estos eventos [14]. El sistema de notificación utiliza esta información.

El sistema SASVi ofrece soporte de asistencia vial a través de notificaciones auditivas cuando existe la posibilidad de la existencia de una irregularidad en el pavimento o cuando se ha rebasado un límite de velocidad para la vía en la cual se transita. Las pruebas desarrolladas para el servicio de asistencia muestran que con una conexión de 1 y 2 Mbps la actualización del reglamento vial en el celular toma entre 1 y 3 segundos. Se comenta que el reglamento vial se simuló ya que hasta donde se conoce no existe un mapa de reglamento vial por zonas urbanas. Respecto al registro de evidencia en formato media se obtuvo que la transferencia de una imagen de $1920 \mathrm{x}$ 
1080 pixeles del celular al Dropbox tardó de 4 a 10 segundos, mientras que la transferencia de videos de 2 minutos tardan entre 1 y 4 minutos, por supuesto, esto depende del ancho de banda disponible en la red. Por otro lado, la binarización de la imagen se desarrolla en promedio en 2 segundos.

\section{Discusión de Resultados}

Para su operación SASVi asume que existe una plataforma digital en la ciudad [15] mediante la cual es posible el intercambio de información entre el teléfono celular del conductor y los servicios digitales en la nube. Limitante que no existe con los agentes de vialidad ya que en la actualidad se hace uso de los servicios $3 \mathrm{G}$ para comunicar las unidades con el centro de mando. Otro factor considerado durante la evaluación del sistema propuesto es la existencia de un reglamento vial sectorizado. Esta es una propuesta a desarrollar ante la dirección de vialidad de la ciudad, la cual podría ajustarse a los esfuerzos iniciados por el gobierno para la sectorización del sistema policial. Con la disponibilidad de contextos viales locales se habilita un intercambio de datos ágil entre los dispositivos móviles y la nube, sin la necesidad de utilizar estrategias de alto costo computacional como las requeridas para la identificación en tiempo real de señalizaciones viales utilizando procesamiento de imágenes [16]. Finalmente, el contexto de seguridad que ofrece SASVi solo considera posibles agresiones de parte del conductor hacia el oficial debido a que el oficial puede como parte de un protocolo de seguridad portar la tecnología necesaria para garantizar la veracidad de los hechos [17]. Por el contrario, un ciudadano al volante puede colocar su teléfono celular en la bolsa de su pantalón, blusa, bolso de mano, compartimiento en la puerta o guantera del auto, lo cual compromete la calidad del audio que proviene de la interacción entre el oficial y el conductor.

\section{Conclusiones y trabajo a futuro}

En este trabajo se ha descrito la plataforma SASVi la cual ofrece servicios de asistencia y seguridad vial. Los servicios de asistencia disponibles permiten notificar al conductor y mantenerlo alerta de las reglas de vialidad aplicables en la zona por la cual transita. Además le va indicando al conductor acerca de las diferentes irregularidades que existen en el pavimento a lo largo de su trayectoria. El servicio de seguridad que se ofrece se basa en el registro de evidencia que puede dar soporte al registro de hechos realizado por el oficial de vialidad y de contar con la silueta del rostro del conductor en caso de que exista algún tipo de agresión contra el oficial de vialidad.

Las pruebas desarrolladas con SASVi son alentadoras y permiten visualizar posibilidades de apoyo para las estrategias de gobierno enfocadas a brindar seguridad y confort a los ciudadanos en las vías de comunicación terrestres. Sin embargo, para poder llevar a SASVi a un nivel de demostración masiva es necesario fortalecer algunos aspectos técnicos de esta plataforma. Primero, en el mejor de los casos el sensor GPS presenta en ocasiones un error de hasta 10 metros, pero en ocasiones 
puede incluso existir pérdida de información geo-espacial por la oclusión con el satélite. Por lo tanto, se debe utilizar información de otros sensores como el acelerómetro y giróscopo para robustecer este servicio. Para reducir el margen de error del proceso de clasificación de baches, topes y bollas se están explorando algoritmos de clasificación como máquinas de soporte de estado y árboles de decisión, además de la integración de técnicas de "crowdsourcing". Se debe adicionalmente, incrementar la calidad de procesamiento para la detección de rostros y posiblemente integrar un proceso automatizado de reconocimiento de los mismos para facilitar la identificación de conductores agresivos. Finalmente, no existen facilidades para notificar al conductor sobre tiempos de traslado desde su ubicación actual hasta su destino ni tampoco opciones de rutas con mejor flujo de tráfico, lo cual puede complementar los servicios presentes en SASVi.

\section{Referencias}

1. Mannering, F. (2009). An empirical analysis of driver perceptions of the relationship between speed limits and safety. Transportation Research Part F: Traffic Psychology and Behaviour, 12(2), 99-106.

2. Bierlaire, M., Chen, J., \& Newman, J. (2010). Modeling route choice behavior from smartphone GPS data.Report TRANSP-OR, 101016, 2010.

3. Yang Guo. (2009). A Mobile Distributed System for Personal Security. Department of information Technology.Institutionen for informationsteknologi.

4. Aldunate, R. G., Herrera, O. A., \& Cordero, J. P. (2013). Early Vehicle Accident Detection and Notification Based on Smartphone Technology. In Ubiquitous Computing and Ambient Intelligence. Context-Awareness and Context-Driven Interaction (pp. 358-365). Springer International Publishing.

5. Eren, H., Makinist, S., Akin, E., \&Yilmaz, A. (2012, June). Estimating driving behavior by a smartphone.In Intelligent Vehicles Symposium (IV), 2012 IEEE (pp. 234-239).IEEE.

6. Kertész, I., T. Lovas, and A. Barsi. "Photogrammetric pavement detection system." The International Archives of the Photogrammetry, Remote Sensing and Spatial Information Sciences 37 (2008).

7. Joubert, D., Tyatyantsi, A., Mphahlehle, J., \& Manchidi, V. (2011). Pothole tagging system.

8. Mednis, Artis, Girts Strazdins, Martins Liepins, AndrisGordjusins, and Leo Selavo. "Roadmic: Road surface monitoring using vehicular sensor networks with microphones." In Networked Digital Technologies, pp. 417-429. Springer Berlin Heidelberg, 2010.

9. Eriksson, Jakob, Lewis Girod, Bret Hull, Ryan Newton, Samuel Madden, and HariBalakrishnan. "The pothole patrol: using a mobile sensor network for road surface monitoring." In Proceedings of the 6th international conference on Mobile systems, applications, and services, pp. 29-39. ACM, 2008.

10. Fazeen, Mohamed, Brandon Gozick, Ram Dantu, MoizBhukhiya, and Marta C. González. "Safe driving using mobile phones." Intelligent Transportation Systems, IEEE Transactions on 13, no. 3 (2012): 1462-1468.

11. Shinar, D., Treat, J. R., \& McDonald, S. T. (1983). The validity of police reported accident data. Accident Analysis \& Prevention, 15(3), 175-191.

12. Harris, D. A. (2010). Picture This: Body-Worn Video Devices (Heads Cams) as Tools for Ensuring Fourth Amendment Compliance by Police. Tex. Tech L. Rev., 43, 357. 
13. Ethem A. (2009). Introduction to Machine Learning. The MIT Press; second edition.

14. Martínez, F., Carlos Gonzalez, L., \& Ricardo Carlos, M. (2014). Identifying Roadway Surface Disruptions Based on Accelerometer Patterns. Latin America Transactions, IEEE (Revista IEEE America Latina), 12(3), 455-461.

15. Ishida, T. (2000). Understanding digital cities. In Digital Cities (pp. 7-17). Springer Berlin Heidelberg.

16. De la Escalera, A., Armingol, J. M., \& Mata, M. (2003). Traffic sign recognition and analysis for intelligent vehicles. Image and vision computing, 21(3), 247-258.

17. Harris, D. A. (2010). Picture This: Body Worn Video Devices ('Head Cams') as Tools for Ensuring Fourth Amendment Compliance by Police. Texas Tech Law Review, Forthcoming. 